\title{
ESTRATÉGIAS METODOLÓGICAS DE EDUCAÇÃO E ASSISTÊNCIA NA ATENÇÃO BÁSICA DE SAÚDE
}

Marta Julia Marques Lopes ${ }^{1}$ João Luis Almeida da Silva ${ }^{2}$

Lopes MJM, Silva JLA. Estratégias metodológicas de educação e assistência na atenção básica de saúde. Rev Latino-am Enfermagem 2004 julho-agosto; 12(4):683-8.

Trata-se de discussão sobre estratégias ou ferramentas metodológico-assistenciais utilizadas na prática em atenção básica de saúde. Baseia-se no diálogo estabelecido entre o que se pensa e o que se desenvolve nos Ambulatórios de Promoção da Qualidade de Vida-APQVs. Esses ambulatórios estão sediados em dois serviços de atenção básica do município de Porto Alegre-RS e seus usuários são, em sua maioria, pacientes adultos e idosos, portadores de doenças de longa duração. A proposta desta discussão é oriunda de um projeto de pesquisa financiado pelo CNPq e integrante da rede temática intitulada: Metodologias de Educação e Assistência na Promoção da Qualidade de Vida. A partir dessa base empírica e conceitual, construíram-se ferramentas metodológicas para o desenvolvimento de consultoria em enfermagem na atenção ambulatorial a indivíduos e grupos. Este artigo propõe-se a apresentar os pressupostos relacionais e operacionais utilizados na assistência nesses serviços.

DESCRITORES: avaliação em enfermagem; cuidados primários de saúde; serviços básicos de saúde; doença crônica

\section{METHODOLOGICAL EDUCATION AND CARE STRATEGIES IN BASIC} HEALTH CARE

This paper discusses methodological and care strategies or tools used in basic health care practice. It is based on the dialogue established between what we think and what we carry out at the Life Quality Promotion Outpatient Centers (APQVS). These centers are located at two basic health care centers in Porto Alegre/RS, Brazil. Its users are mostly adult and elderly patients with long-term illnesses. The proposal of this discussion arose from a research project financed by the Brazilian Council for Scientific and Technological Development - CNPq, and integrates a thematic network called Education and Care Methodologies to Promote Life Quality. Starting from this empirical and conceptual base, methodological tools were built to develop nursing consulting services in outpatient health care to individuals and groups. This article aims to present relational and operational concepts used in care at these services.

DESCRIPTORS: nursing assessment; primary health care; basic health services; chronic disease

\section{ESTRATÉGIAS METODOLÓGICAS DE EDUCACIÓN Y ASISTENCIA EN LA} ATENCIÓN BÁSICA DE SALUD

Se trata de una discusión sobre las estrategias o herramientas metodológicas-asistenciales utilizadas en la práctica de atención básica de salud, basada en el diálogo establecido entre lo que pensamos y lo que desarrollamos en los Ambulatorios de Promoción de Calidad de Vida - APQVs. Esos ambulatorios están localizados en dos servicios de atención básica en Porto Alegre/RS, Brasil. La mayoría de sus usuarios son pacientes adultos y ancianos portadores de enfermedades de larga duración. La propuesta de esta discusión se origina en un proyecto de investigación financiado por el Consejo Brasileño de Desarrollo Científico y Tecnológico y hace parte de la red temática titulada: Metodologías de Educación y Asistencia en la Promoción de la Calidad de Vida. A partir de esa base empírica y conceptual, se construyeron las herramientas metodológicas para el desarrollo de consultoría en enfermería en la atención clínica a individuos y grupos. Este artículo se propone presentar los presupuestos relacionales y operacionales utilizados en la asistencia en esos servicios.

DESCRIPTORES: evaluación en enfermeria; atención primaria de salud; servicios básicos de salud; enfermedad crónica

\footnotetext{
${ }^{1}$ Doutor em Sociologia, Professor Titular, e-mail: marta@enf.ufrgs.br; ${ }^{2}$ Enfermeiro, Mestrando do Curso de Pós-Graduação em Enfermagem, Bolsista do Programa CAPES, e-mail: joao.almeida@ufrgs.br. Universidade Federal do Rio Grande do Sul
} 
Essa perspectiva, acredita-se, impõe cada vez

Este artigo propõe-se a apresentar e discutir noções conceituais, aspectos relacionais e operacionais para a estruturação de ferramentas metodológicoassistenciais e educativas, direcionadas à Atenção Básica de Saúde. É resultado de quatro anos de pesquisadesenvolvimento e assistência, motivado pela idéia de investir no caráter coletivo, multidisciplinar, interdependente e complementar dessas práticas. As reflexões situam-se no diálogo estabelecido entre o que se pensa e o que se desenvolve nos Ambulatórios de Promoção da Qualidade de Vida-APQVs*. Esses ambulatórios estão sediados em dois serviços de atenção básica do município de Porto Alegre-RS e seus usuários são pacientes adultos e idosos, portadores de doenças de longa duração, em sua maioria.

A modalidade de pesquisa-desenvolvimento, muito utilizada em estudos comunitários, sustenta a ação nos APQVs. Essa forma de aproximação do campo de atuação tem, sob tal ponto de vista, o mérito de responder às investigações com conhecimento de causa, além de testar modelos teóricos advindos da análise diagnóstica, com rigor necessário à implantação das ações concretas. Trata-se, portanto, de modalidade de pesquisa-ação onde a implantação de estratégias assistenciais, na forma de protocolos, é acompanhada de procedimentos de investigação e validação. A transposição do conceito de diagnóstico para o domínio das ciências humanas e sociais permite analisar contextos e sistemas organizados. Nesse sentido, significa conhecimento novo, junção de dados confrontados, sintetizados, articulados que permitem leitura global da realidade estudada. O conhecimento gerado pelo diagnóstico permite adaptar decisões, planejar ações adequadas ao contexto de indivíduos e coletivos ${ }^{(1-}$ 2).

A partir desses subsídios técnicos, há reflexões sobre a intervenção educativa e assistencial através da estratégia da Consulta de Enfermagem. Parte-se, também, da necessidade do investimento em novos referenciais para o embasamento de práticas que privilegiem a noção de saúde e qualidade de vida. No centro das preocupações está a adoção da visão integral dos sujeitos assistidos e do trabalho complementar e interdependente das práticas dos profissionais nesse campo, propondo abordagens no plano individual e coletivo. mais a urgência de aprimoramentos e a prática de ações que implementem novos protocolos de atenção em saúde, adaptados às novas realidades e ao perfil epidemiológico atual das demandas dos serviços.

Nesse sentido, a elaboração de protocolos assistenciais como ferramentas metodológicas para a prática de enfermagem ambulatorial e, particularmente, para a estratégia de consulta, constitui procedimento importante para o aperfeiçoamento das ações na Atenção Básica. Os protocolos, ou acordos técnicos, baseados na atualização teórica e nos avanços do conhecimento da área, proporcionam subsídios necessários à adequação científica e tecnológica dos processos terapêuticos. Também conferem certa homogeneidade às condutas técnicas que evitam disparidades e facilitam o cotidiano do trabalho ambulatorial, tornado-o resolutivo e competente.

Estudos recentes, desenvolvidos por enfermeiras(os), consideram os protocolos como ferramentas que auxiliam na sistematização da consulta, subsidiando o desenvolvimento de lógica técnico-científica que necessita ser atualizada no sentido do aprimoramento do raciocínio clínico e educativo. Esses estudos demonstram inclusive a eficácia da Consulta de Enfermagem através de ensaios clínicos e multicêntricos, os quais se baseiam no impacto e eficácia mensuráveis através dos resultados clínicos, decorrentes da ação de diferentes profissionais trabalhando com uma base técnica comum $^{(3-4)}$.

Experiências locais em serviços de Atenção Básica de Saúde têm buscado, na elaboração de protocolos, maior resolutividade e o fortalecimento da participação de enfermeiras em atividades preventivas, curativas, educativas, diagnósticas, terapêuticas e de reabilitação ${ }^{(5-6)}$

Do ponto de vista da enfermagem, a legislação atual do exercício profissional, ao referir-se à "programação de enfermagem", salienta a prescrição da assistência como prerrogativa da(o) enfermeira(o), incluindo ações técnicas como consultorias, auditorias e emissão de pareceres sobre matéria de enfermagem, além da Consulta de Enfermagem ${ }^{(7)}$. A Consulta de Enfermagem, nesse caso, é a ação de atender indivíduos em consultório, executada por profissionais de enfermagem, capacitados

\footnotetext{
* Os APQVs inserem-se em um projeto de pesquisa-desenvolvimento, financiado pelo CNPq, inserido na Rede Temática: Metodologias de Educação e Assistência na Promoção da Qualidade de Vida. Os pesquisadores e enfermeiros assistenciais são oriundos da EENF/UFRGS, Grupo Hospitalar Conceição e Programa Saúde da Família de Porto Alegre, coordenados pela Prof프를 Marta Julia Marques Lopes
} 
para tal. A consulta, enquanto ato de consultar ou ser consultado, caracteriza o ato de pedir ou dar conselho, opinião, instrução, parecer. Essa definição constante do dicionário da língua portuguesa ${ }^{(8)}$, refere ainda que "consultor é aquele que dá ou pede conselho; aquele que consulta, examinando...aquele que dá pareceres acerca de assunto de sua especialidade...em lugar ou casa onde se dão consultas". A essas definições agrega-se a noção de estratégia metodológica para o exercício profissional de enfermagem. A utilização da consulta, em seu formato de ferramenta metodológica para sistematização da assistência profissional, é variável segundo as bases e prerrogativas técnico-profissionais das diferentes profissões no campo da saúde. A consulta, no entanto, não é em si prerrogativa profissional exclusiva, podendo ser exercida em seu sentido mais amplo, em diferentes áreas do saber profissional e, mesmo, como estruturante das relações em sociedade simplesmente.

A adoção da consultoria especializada, na forma da Consulta de Enfermagem, tem sido relatada na bibliografia nacional e implantada nos serviços desde a década de 60 , principalmente na saúde coletiva ${ }^{(9-10)}$. No entanto, não é intenção discutir, nesse momento, aspectos históricos do conhecimento já produzido, mas, sim, avançar sobre um outro ângulo através de algumas referências. Esse "outro ângulo" considera a construção de saberes práticos que viabilizem novas ações, troca de conhecimentos e tecnologias em saúde.

Objetiva-se, portanto, discutir quais práticas se adequam e dão sustentação a essas idéias e como essas idéias podem influenciar continuamente essas práticas. Assim, a Consulta de Enfermagem, como referida na legislação profissional, como estratégia metodológica de educação e assistência é o foco desta reflexão.

\section{A CONSULTA COMO É PENSADA E DESENVOLVIDA NO AMBULATÓRIO DE PROMOÇÃO DA QUALIDADE DE VIDA}

A idéia de que o processo saúde-doença é resultante das situações de vida dos indivíduos e coletivos e que essas situações são construídas nas relações que se estabelecem na sociedade, sendo, portanto, históricas e passíveis de serem apreendidas concretamente, é parte importante do ideário constitutivo de práticas de consultório.
A noção de situações de vida, portanto, incorporada a partir da contribuição das ciências humanas e sociais, serve como aliada para que se possa compreender melhor a complexidade dos sujeitos e dos coletivos aos quais são dirigidas as intervenções profissionais.

Nesse caso, agregam-se novas perspectivas na intervenção em saúde que não se limitam aos aspectos técnicos. É no reconhecimento desses limites que são pensadas as atuais formas de intervir em saúde, opondo integralidade à fragmentação das propostas do modelo biologicista dominante.

A proposta metodológica de educação e assistência que se preconiza para a estratégia de consulta, baseia-se em abordagem abrangente e dinâmica, capaz de desenvolver visão multidimensional dos indivíduos, famílias e comunidades. Essa visão busca entender os processos de interação com fatores socioambientais que compõem o processo saúde-adoecimento, ou que servem de estímulo ao processo de autocuidado ${ }^{(11)}$.

Nesse sentido, a consulta que se propõe agrega as seguintes questões: como ajudar as pessoas? como a relação de consulta pode ser competente para os dois lados?

Para que tais questões sejam respondidas, é necessário adequar a conduta dos profissionais enquanto "consultores" centrados em si mesmos, pois, freqüentemente, esforçam-se para responder às próprias necessidades e não aquelas dos pacientes. Geralmente, preocupam-se mais com o racional do que com o emocional.

Examinando essa conduta, dominante nas atitudes de consultório e nas práticas em serviços de Atenção Básica e comunitários, tenta-se, criticamente, discutir e adotar perspectivas inovadoras que considerem tanto os aspectos técnicos (e operacionais) envolvidos no processo de consulta, como os relacionais, fundamento das relações humanas. Esses aspectos foram desenvolvidos por essa equipe ${ }^{(12)}$ e consideram que o relacional e o técnico são componentes da ação de cuidar e que o relacional é o que singulariza a ação de enfermagem.

Pressupostos relacionais na estratégia de consulta

Os pressupostos relacionais, construídos a partir de experiências de consultório, consideram: a escuta ativa 
- que é o engajamento no sentido de estar "com", tentando desenvolver no outro o sentimento de "ser compreendido"; a aceitação do outro, além do que se pensa ou do que ele diz, que permite desenvolver uma aceitação compreensiva e não normativa; a empatia, que é o esforço para compreender os sentimentos das outras pessoas e não apenas suas idéias; a confiança, que é construída no desenrolar da relação de consulta e se desenvolve a partir de uma tomada de consciência das próprias emoções do profissional e do manejo adequado na direção da ação terapêutica e, por fim, a ausência de julgamento que, acredita-se, pode tornar-se forte aliada no sentido de que julgar negativamente uma conduta do paciente nem sempre resulta no desencadeamento de atitude adequada e de adesão à terapêutica $^{(12-13)}$.

Nesse sentido, o desenvolvimento da capacidade de empatia merece destaque. Essa capacidade requer esforço do terapeuta, pois certas situações de consulta mobilizam sentimentos e inquietudes. O esforço está em não reagir às situações adversas no lugar do paciente e, sim, em estar próximo e ajudá-lo no enfrentamento e reformulação do problema que se apresenta. Nesse caso, é necessário ter presente que a atitude de mudança é resultado da reformulação que o paciente elabora sobre sua situação. A importância da empatia na relação de cuidado reflete-se positivamente na comunicação profissional-paciente e facilita o processo dinâmico de negociação terapêutica. O estímulo ao reconhecimento das próprias qualidades e capacidades é outro aspecto a ser desenvolvido ativamente pela(o) terapeuta.

É preciso que se tenha claro que a doença produz efeitos sobre as representações de si e ataca a autoimagem e a auto-estima. A recuperação desses aspectos leva à confiança na aquisição de condutas e de capacidades para enfrentar os desafios vividos na doença.

Partindo desses pressupostos, entende-se que o "projeto terapêutico", nascido dessa interação profissionalpaciente, torna-se instrumento de educação em saúde, competente e resolutivo para "os dois lados".

O plano assistencial buscará, dessa forma, estabelecer prioridades aos problemas e/ou fatores nocivos (danos) identificados, especificar os resultados que se espera que o paciente alcance e construir as ações específicas de intervenção traçadas junto com ele. Acredita-se que durante o processo de interação é necessário negociare/ou fazer contratos para uma efetiva adesão à terapêutica. Ou seja, é preciso procurar, ao longo da consulta, desenvolver uma aliança terapêutica, ir completando (relembrando) as questões já tratadas anteriormente para seguir adiante ou retomar as discussões e metas em consultas periódicas ${ }^{(3-9,11-12,14)}$.

Pressupostos operacionais na estratégia de consulta

Esse projeto terapêutico organiza-se, quanto aos aspectos técnicos, no que se chama pressupostos operacionais que são: a) o tempo dedicado a cada indivíduo, nesse caso sendo 45 minutos para o primeiro contato e em torno de 30 minutos para os subseqüentes; b) o acolhimento e a escuta, embasados nas concepções de que acolher não se resume a uma recepção cordial e a escuta é uma atitude ativa; c) a utilização de ferramentas técnicas para o exame físico para detecção dos aspectos evolutivos das doenças de longa duração; d) a análise interativa, que é a confrontação de elementos centrada na comunicação (verbal e não-verbal) e na ênfase aos aspectos da educação em saúde com interfaces e participação multidisciplinar e interdisciplinar; e) a elaboração conjunta e negociada do plano terapêutico com medidas educativas e de auto-cuidado; f) a construção conjunta do vínculo com o serviço e o terapeuta, por meio de agendamento periódico e encaminhamentos, considerando-se aspectos técnicos, necessidades e motivações dos indivíduos; g) o acompanhamento evolutivo individual ou em grupos.

Em síntese, pode-se dizer que a proposta dedicase a avaliar e permitir ao indivíduo (paciente) o constante reexame de suas expectativas e modos de superação e adaptação ao seu problema de saúde, explorando conjuntamente o sentido que o mesmo dá a esse problema ou doença.

Já os contatos ou consultorias de acompanhamento dos pacientes, precisam considerar dois níveis de intervenção ou avaliação técnica: o nível psicológico e o instrumental. Esses níveis cumprem a importante função da "presença ativa", ou seja, o paciente sabe que pode contar com alguém naquele local no momento em que necessita ou com uma regularidade geradora de segurança.

A partir da experiência de consultório, concluímos que no nível psicológico (tendo claro os limites técnicos a esse propósito), é essencial desenvolver habilidades de escuta e troca centradas na trajetória emocional de cada pessoa. Essa atitude do terapeuta permite reduzir o impacto de condutas ou efeitos radicais ou traumáticos 
da terapêutica, incluindo as mudanças comportamentais que exigem muito esforço dos pacientes. Essas mudanças, não raro, produzem efeito desorganizador na vida deles. Assim, uma dinâmica baseada na escuta e na ação interativa negociada permite, sob esse ponto de vista, a mudança e adaptação necessárias ao enfrentamento de novas situações e realidades.

O nível instrumental da avaliação possibilita a estruturação de proposições renovadas a cada consulta e a aprendizagem necessária à manutenção da independência do paciente. Ou seja, a adoção de novas atitudes que requeiram conhecimento e habilidades no manejo de novos instrumentos para o autocuidado, por exemplo. São freqüentes, em consultório, os questionamentos sobre a reorganização da vida dos pacientes frente a novas condutas ou às limitações impostas pelo problema, ou processo patológico, as quais, muitas vezes, são restritivas, incapacitantes e geradoras de dependência.

As readequações, do ponto de vista das necessidades comunicacionais, também são freqüentes nas queixas dos pacientes. Nas relações em sociedade, perda ou redução da audição ou da visão e mesmo efeitos da depressão são aspectos que precisam ser considerados e "negociados" na construção de soluções ou atitudes de mudança progressiva e/ou adaptação. Nesse sentido, pensa-se que a articulação entre esses níveis facilita a adesão progressiva e constante às proposições terapêuticas e educativas.

Uma terapeuta francesa ${ }^{(14)}$ tece considerações sobre o que chama de entrevista na relação de consulta. Confronta-se essa entrevista proposta com a prática aqui mencionada, reelaborada e adotada no sentido de aperfeiçoar essa relação. Diz a autora que, para o acompanhamento dos pacientes e, aqui se acrescenta, para "responder à interatividade", necessitam-se atitudes e abordagens como segue: acolher as reações verbais e não-verbais do paciente; explorar junto com o paciente seus medos, incertezas, expectativas e esperanças; explorar com o paciente o impacto da doença e de seus tratamentos (imediato e para a vida); identificar as novas necessidades e as mudanças ligadas à nova condição somática; avaliar a capacidade de sustentação da família; ouvir e ajudar o paciente a elaborar ou resolver os problemas concretos que aparecem na evolução da doença e do tratamento, como emprego, auxílio para a realização de atividades cotidianas, problemas financeiros, a relação com os outros, entre muitas outras questões.
Uma dificuldade concreta é mudar a atitude no sentido do "deixar-se cuidar". Depara-se com o medo da dependência como fator de dificuldade em situações de doença de longa duração que afetam ainda mais a qualidade de vida.

Considera-se também que os processos comunicacionais, verbais e não-verbais, devem ser aperfeiçoados e adaptados às características dos indivíduos e necessidades identificadas. Para tal, um guia de entrevista no momento da consultoria objetiva facilitar a exploração dos fatos e sentimentos envolvidos, sem esquecer os diferentes aspectos que podem aflorar.

Preferentemente, conforme sugere a mesma autora $^{(14)}$, a entrevista deve ser construída com questões que não podem ser respondidas por "sim" ou por "não", mas por questões que estimulem o paciente a exercitar uma elaboração pessoal dos fatos ocorridos. O exercício conjunto de (re)formulação da descrição é facilitador no sentido da compreensão do que se quer dizer ou expressar na forma de sentimentos.

Relativamente aos sentimentos, é comum o negativismo face aos problemas tidos como difíceis ou insolúveis em situações de consultoria. Essa situação é melhor trabalhada se esse sentimento é devolvido ao paciente (como num espelho, diz a autora). Essa atitude é uma tentativa de tornar o paciente capaz de identificar seus problemas, compreendê-los e transformá-los. Essa atitude da(o) terapeuta permite ao paciente focalizar seu problema e se reapropriar dele, facilitando assim sua resolução.

Essas condutas técnicas (ou protocolos) reconhecidas cientificamente como, por exemplo, um guia ou um histórico para a entrevista na relação de consulta adotados a partir de pressupostos teórico-filosóficos norteadores - facilitam e tornam mais competente (para os dois lados) o trabalho assistencial.

Esses processos comunicacionais (com caráter técnico e relacional) como aqui se prefere chamar, devem incluir a adequação com criatividade e individualização do seu uso. Desenhos, imagens, palavras, atividades lúdicas, entre outras, são instrumentos importantes na eficácia das condutas propostas e devem ser realizadas conjuntamente terapeuta-paciente ou terapeuta-grupo.

Enfim, a partir dessa compreensão complexa da relação terapêutica, o conteúdo desses instrumentos de abordagem necessita explorar com o paciente os fatos que atestam sua forma de integrar a terapêutica 
comportamental, ou o tratamento propriamente dito, na sua vida cotidiana. Então, torna-se possível analisar expectativas, resultados e impacto físico e mental bem como novas necessidades surgidas.

\section{CONSIDERAÇÕES FINAIS}

Atualmente, ao se experienciar essa forma de fazer técnico-profissional, consolidam-se alguns caminhos metodológicos possíveis de auxiliar a responder dinamicamente às situações que se apresentam na prática ambulatorial de Atenção Básica de Saúde. Ao apresentar esses pressupostos à comunidade científica de

\section{REFERÊNCIAS BIBLIOGRÁFICAS}

1. Dufumier M. La recherche développement. Le projets de développement agricole. Paris (FR): CTA-KARTHALA; 1996. 2. Gonnet F. L'hôpital en question(s): un diagnostic pour améliorer les relations de travail. Paris (FR): Lamarre; 1992. 3. Ferreira SRS. História e avançar no trabalho de enfermagem na Divisão de Saúde Comunitária do Grupo Hospitalar Conceição. Momento e Perspect em Saúde 1996; 9(2).

4. Hidalgo PLP, Fernández FPG. Aténción por enfermeras a pacientes com enfermedades leves en consultas de Atención Primaria. Index de Enfermeria 2001 primavera-verano; 10(32):57-9.

5. Prefeitura Municipal de Porto Alegre. Procedimentos e ações em saúde para o profissional enfermeiro. Porto Alegre (RS): Secretaria Municipal de Saúde/Assessoria de Planejamento; 2002.

6. Ambulatórios do HNSC e SSC/GHC. Rotinas de prescrição, transcrição de medicamentos e procedimentos para enfermeiras dos programas de saúde pública. Porto Alegre (RS): Grupo Hospitalar Conceição; 1993.

7. Conselho Regional de Enfermagem do Rio Grande do Sul (RS). Legislação (Lei no 7498, de 25 de junho de 1986 e Decreto no 94406/87, de 8 de junho de 1987). Porto Alegre (RS): Conselho Regional de Enfermagem/RS; 2001.

8. Ferreira $\mathrm{ABH}$. Novo Dicionário da Língua Portuguesa. $2^{a}$ ed. Rio de Janeiro (RJ): Nova Fronteira; 1986.

9. Adami NP, Franco LHRO, Brêtas ACP, Ransan LMO, Pereira AL. Características básicas que diferenciam a consulta de enfermagem da consulta médica. Acta Paul Enfermagem 1989; 2(1):9-13.

10. Vanzin AS, Nery MHS. Consulta de enfermagem: uma necessidade social? Porto Alegre (RS): RM\&L; 1996.

11. Vanzin AS, Nery MHS. Consulta de enfermagem: uma necessidade social? Porto Alegre (RS): RM\&L; 2000.

12. Lopes MJM, Silveira DT, Ferreira SR. Educação em saúde nas doenças crônico-degenerativas e a promoção da qualidade de vida: relato de experiência. Estud Interdisc Envelhec 1999; 2:121-30.
Enfermagem, salientam-se as convicções sobre o caráter interdependente e complementar da ação terapêutica em saúde. Nesse sentido, entendem-se que essa perspectiva pode adequar-se a outras práticas que se proponham a considerar a integralidade dos sujeitos assistidos.

A receptividade e o acompanhamento dos resultados terapêuticos têm mostrado efeitos na criação de vínculo dos usuários com os profissionais e serviços, aumento da adesão às terapêuticas (tanto medicamentosas como comportamentais), valorização e melhora da auto-estima dos sujeitos, estímulo e motivação ao autocuidado. Esses aspectos estão sendo monitorados para avaliação dos resultados e da efetividade terapêutica do serviço para posterior publicação.

13. Lopes MJM. Quando a voz e a palavra são atos terapêuticos: a interação individual e coletiva nas palavras cotidianas do trabalho de enfermagem. In: Waldow VR, Lopes MJM, Meyer DE, organizadoras. Maneiras de cuidar, maneiras de ensinar. Porto Alegre (RS): Artes Médicas; 1995. p. 153-88. 14. Tourette-Turgis C. La retinite à CMV- Guide de counseling. Paris (FR): Comment Dire/Roche; 1996. 